\title{
Machine Learning and Urban Drainage Systems: State-of-the-Art Review
}

\author{
Soon Ho Kwon ${ }^{1}$ (D) and Joong Hoon Kim ${ }^{2, *(\mathbb{D})}$ \\ 1 Future and Fusion Laboratory of Architectural, Civil and Environmental Engineering, Korea University, \\ Anamdong, Seongbukgu, Seoul 02841, Korea; rnjstnsgh90@gmail.com \\ 2 School of Civil, Environmental and Architectural Engineering, Korea University, Anamdong, Seongbukgu, \\ Seoul 02841, Korea \\ * Correspondence: jaykim@korea.ac.kr; Tel.: +82-2-3290-3316
}

\section{check for}

updates

Citation: Kwon, S.H.; Kim, J.H. Machine Learning and Urban Drainage Systems: State-of-the-Art Review. Water 2021, 13, 3545. https://doi.org/10.3390/w13243545

Academic Editor: Zheng Duan

Received: 30 September 2021 Accepted: 8 December 2021 Published: 11 December 2021

Publisher's Note: MDPI stays neutral with regard to jurisdictional claims in published maps and institutional affiliations.

Copyright: (c) 2021 by the authors. Licensee MDPI, Basel, Switzerland. This article is an open access article distributed under the terms and conditions of the Creative Commons Attribution (CC BY) license (https:/ / creativecommons.org/licenses/by/ $4.0 /)$.

\begin{abstract}
In the last decade, machine learning (ML) technology has been transforming daily lives, industries, and various scientific/engineering disciplines. In particular, ML technology has resulted in significant progress in neural network models; these enable the automatic computation of problemrelevant features and rapid capture of highly complex data distributions. We believe that ML approaches can address several significant new and/or old challenges in urban drainage systems (UDSs). This review paper provides a state-of-the-art review of ML-based UDS modeling/application based on three categories: (1) operation (real-time operation control), (2) management (flood-inundation prediction) and (3) maintenance (pipe defect detection). The review reveals that ML is utilized extensively in UDSs to advance model performance and efficiency, extract complex data distribution patterns, and obtain scientific/engineering insights. Additionally, some potential issues and future directions are recommended for three research topics defined in this study to extend UDS modeling/applications based on ML technology. Furthermore, it is suggested that ML technology can promote developments in UDSs. The new paradigm of ML-based UDS modeling/applications summarized here is in its early stages and should be considered in future studies.
\end{abstract}

Keywords: machine learning; urban drainage systems; flood-inundation prediction; flood pattern recognition; pipe defect detection; real-time operation control

\section{Introduction}

Urban drainage systems (UDSs) are used in urban infrastructure to drain rainwater and/or used water from a system without causing floods. In this regard, high-dimensional simulations for UDS modeling (i.e., flood-inundation mapping) is typically performed, and two-dimensional (2D) hydrodynamic/hydraulic simulation models should be considered [1,2]. Two-dimensional models have been widely used to compute complex floodinundation dynamics and hydrological processes $[3,4]$. However, the function evaluations in various UDS problems (e.g., real-time flood-inundation forecasting and real-time operation control), generally conducted using 2D, demand high computational power because of the simulation times involved for rainfall-runoff and pipe network hydraulics [5-8]. Therefore, it is challenging to utilize a physically based hydrodynamic/hydraulic model (i.e., 2D model) for real-time UDS modeling.

This significant limitation can be overcome by considering machine learning (ML) technology. ML technology is transforming daily lives, industries, and science/engineering disciplines [9]. ML technology enables computers to learn and function without explicit instructions and programming, but with patterns and inferences extracted from data [10]. Currently, ML technology (e.g., regression, classification, and clustering) is being replaced with new ML technologies, such as deep learning (DL) approaches [11]. These approaches primarily include big data, which are high-dimensional datasets in sensor-based measurements [12-14]. 
Recently, DL approaches have been widely used across various science/engineering disciplines. In fact, they have been improved more actively than ML approaches [15]. DL approaches are defined as a type of ML technology based on neural networks, in which multiple layers of processing are used to extract progressively higher-level features from a significant amount of data [16]. DL approaches represent a higher level from the underlying datasets [17]. Furthermore, DL approaches are typically more immune to raw and noisy data because they allow practical features to be extracted without being explicitly indicated [18].

DL approaches demonstrate the potential to be applied and utilized in UDSs owing to their ability to address problems in various science/engineering disciplines [19]. Prior to the introduction of ML technology, a physically based model was widely utilized to demonstrate the system characteristics of hydrodynamics/hydraulics in UDSs. Previous UDS studies pertaining to ML technology, including DL approaches, should be revisited and summarized to provide recommendations for the development of UDSs. Herein, a comprehensive review of UDS studies is provided, and potential research topics pertaining to ML applications are recommended. It is noteworthy that this review paper focuses on the aspects of operation, management, and maintenance in UDS studies based on ML technology.

Herein, comprehensive reviews and recommendations are presented in four sections: first, the review methodology of the UDS studies revisited (with respect to operation, management, and maintenance) is described in Section 2. Subsequently, the concept and overview of ML technology are described (Section 3). The objective of this paper is to summarize previous UDS studies pertaining to ML-based modeling/application based on three research categories: (1) operation (real-time operation control), (2) management (flood-inundation prediction), and (3) maintenance (pipe defect detection) (Section 4). Subsequently, some potential issues and future directions are recommended for each of the three research topics to advance UDS modeling/application based on ML approaches (Section 5). ML-based UDS modeling enables ML approaches to promote developments in UDSs.

\section{Review Methodology}

Herein, UDS studies are reviewed comprehensively with respect to operation, management, and maintenance. In this regard, the Web of Science and Google Scholar was used to obtain relevant literature from a wide and extensive web space. The literature identified from the web space (e.g., Web of Science, Scopus, and Google Scholar) was filtered by inspecting the title, summary, and methodology using keywords of "machine learning" and "urban drainage systems". In the selected literature, studies that clearly proposed and/or highlighted ML-based UDSs based on operation, management, and maintenance were selected. Finally, 16 studies were selected and summarized, including five UDS operation studies, six management studies, and five maintenance studies. All selected studies were published after 2018 (note: all studies included the recent trend), even though the publication time for all studies was not customized.

\section{Machine Learning (ML) Technology}

In this section, specific approaches focusing on UDS studies (e.g., operation, management, and maintenance) based on ML technology are summarized. In particular, traditional ML technology (e.g., random forest, support vector machine, and K-nearest neighbor algorithm) has not been widely used with respect to operation, management, and maintenance in UDS studies. Rather than predicting and/or classifying values or factors, most of the studies focused on clustering and/or reducing dimensions on a predefined dataset. Such techniques are not suitable for UDS studies with respect to operation, management, and maintenance.

Therefore, we considered only specific approaches (e.g., artificial neural networks (ANNs), convolutional neural network (CNNs), recurrent neural networks (RNNs), and 
deep q-networks (DQNs)) that are widely used in the operation, management, and maintenance of UDSs. All studies based on ML technology considered various evaluation criteria to verify the model performance. The representative evaluation criteria used in UDS studies are as follows: (1) root-mean-square error, (2) coefficient of determination, and (3) Nash-Sutcliffe efficiency.

\subsection{Overview}

Artificial intelligence (AI) is realized using a specific machine or system based on ML technology. ML technology involves identifying a set of rules and characteristics hidden in the machine via direct training based on data. In other words, computers perform evaluation and prediction from the learning process based on a specific type of data possessed by humans. Problems that can be solved using ML technology can be generally classified into (1) problems requiring classes of specified data to be distinguished (pattern recognition), (2) problems requiring the estimation of certain consecutive values (regression), and (3) functional approximation.

In addition, ML technology based on learning methods can be classified into three types of learning: (1) supervised learning, (2) unsupervised learning, and (3) reinforcement learning. First, supervised learning is a technique that provides data and labels (answers) for a problem. Humans wish to obtain solutions using learning data, and then estimate and provide appropriate labels when inputting new unlabeled data. This technique is primarily used for object recognition, label estimation probability, and regression analysis. Unsupervised learning is a technique where unlabeled data are received and new characteristics, patterns, and classes are identified from the data via learning. It is primarily used to solve problems such as clustering, feature extraction, and dimensionality reduction. Finally, reinforcement learning is a method in which an agent defined within an environment recognizes the current state and selects an action or sequence of actions that maximizes compensation among selectable behaviors. ML technology allows different problems to be solved based on the learning type, as summarized and presented in Figure 1.

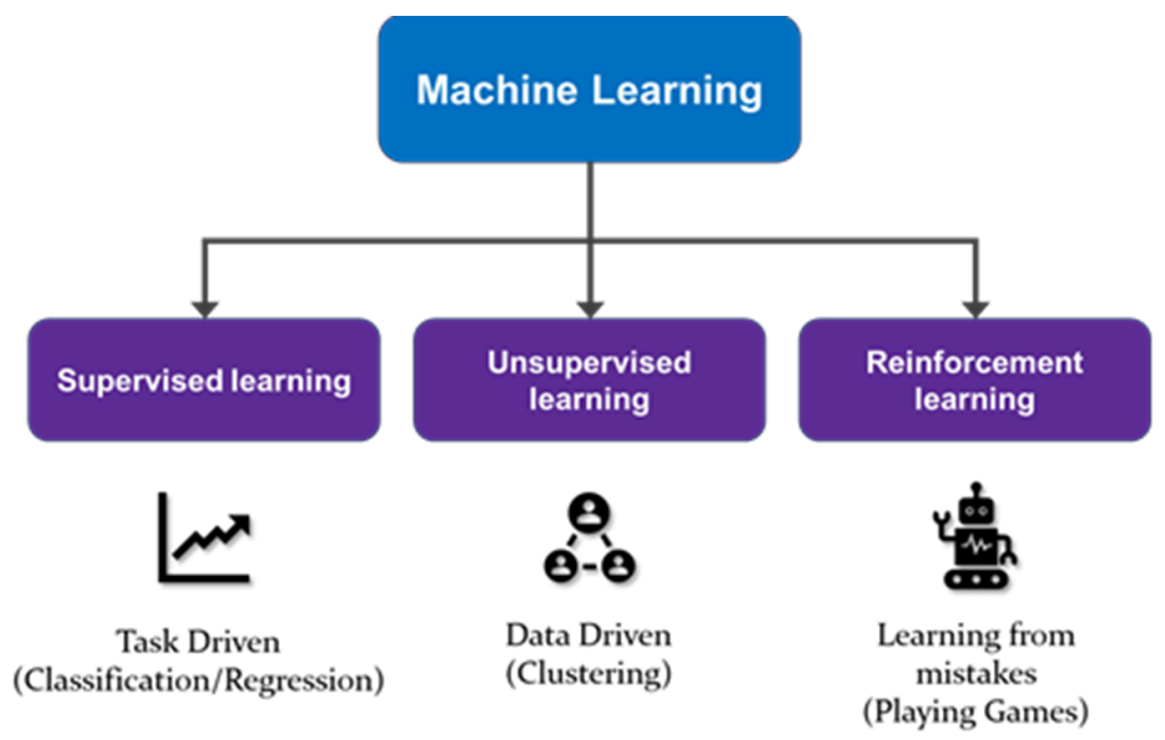

Figure 1. Overview of machine learning (ML) technology.

The following section describes the characteristics, architecture design, and learning method of the widely used neural networks in ML technology. It is noteworthy that the neural networks described herein include only ANNs, CNNs, RNNs, and DQNs. 


\subsection{Artificial Neural Networks (ANNs)}

An ANN, which is a conventional neural network, is a computing approach inspired by biological neural networks that comprise animal brains [20]. The ANN is designed in the form of a network by combining synapses. It comprises an input layer, a hidden layer, and an output layer, and each circle in the ANN is a neuron (Figure 2a). The hidden layer is composed of either a single layer (only one layer) or multiple layers (more than two layers). An activation function is performed in the hidden layer, which transforms an input into an output. The activation function activates the sum of signals.

(a) ANN

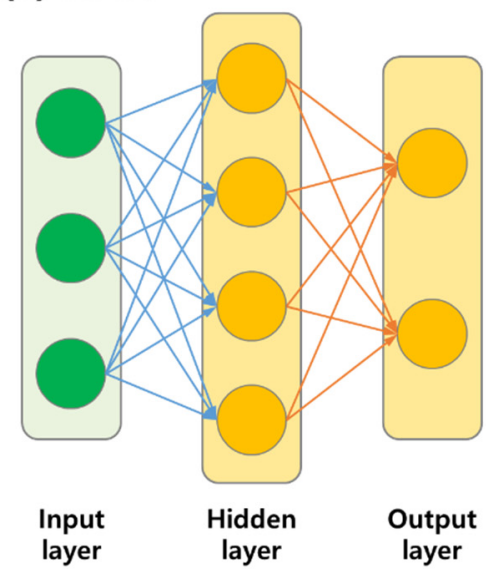

\section{(b) RNN}

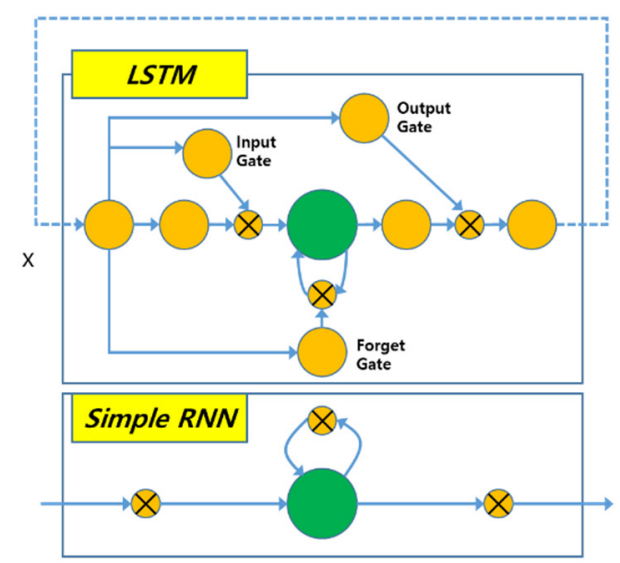

(c) CNN

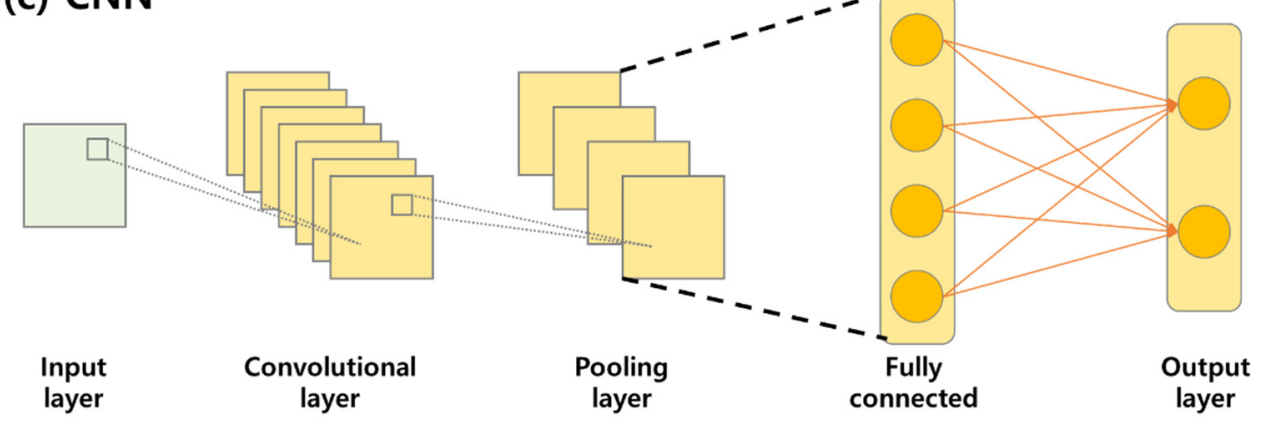

\section{(d) DQN}

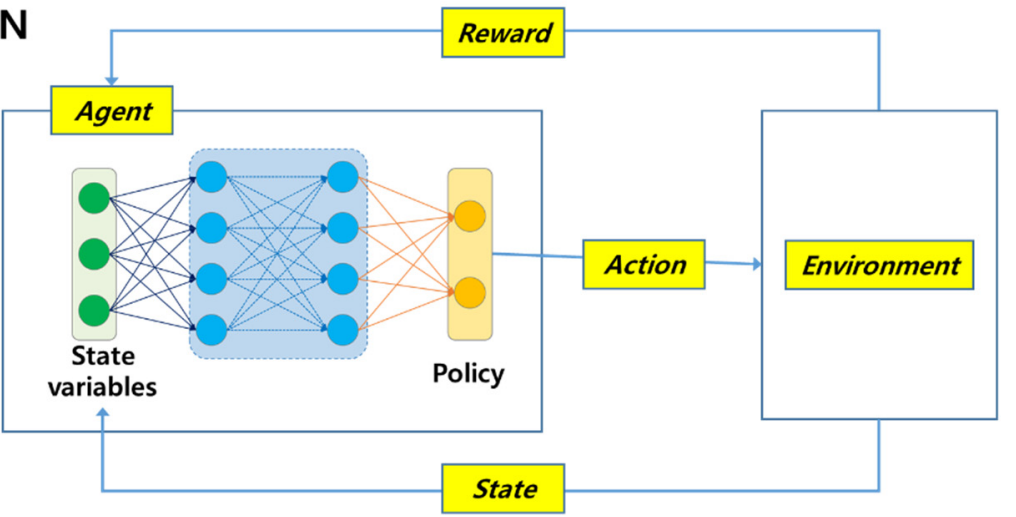

Figure 2. Architecture of several neural network structures. (a) ANN; (b) RNN (including LSTM); (c) CNN; (d) DQN. 


\subsection{Recurrent Neural Networks (RNNs)}

The RNN is a type of neural network whose connection between nodes exhibits a recurrent structure (Figure 2c). This structure allows the state to be stored inside the circulating neural network model such that the characteristics can be dynamically analyzed over time [21-23]. In particular, unlike the ANN of the existing ML technology, this circulation structure may process a sequence-type input based on internal memory. In general, it is primarily used for voice signal processing and language string processing. RNNs can be categorized into different networks (the representative network is long short-term memory, LSTM).

LSTM is suitable for classification, processing, and prediction based on time-series data because unknown delays may occur between important events in a time series [24]. LSTM is developed to alleviate gradient loss problems that can occur when training conventional RNNs. LSTM units consist of a cell, an input gate, an output gate, and a forget gate, and their LSTM units determine unnecessary information or remember information.

\subsection{Convolutional Neural Networks (CNNs)}

A CNN is a type of DL approach for processing data that exhibit a grid pattern, such as images. It is inspired by the organization of the animal visual cortex $[25,26]$ and constructed based on adaptively and automatically learned spatial hierarchies of features, from low- to high-level patterns [27]. CNNs are mathematical constituents that are generally composed of three types of layers: convolution, pooling, and fully connected layers (Figure 2b).

The first two layers (i.e., convolution and pooling layers) perform feature extraction, whereas the third layer (a fully connected layer) maps the identified features into the final output, such as classification [28,29]. The convolution layer, which is critical to the $\mathrm{CNN}$, is composed of a series of mathematical computations, such as convolution. In digital image processing, a pixel value is used in a 2D grid, and a small grid of parameters (i.e., kernel), which is an optimizable feature extractor, is applied to each image position. Therefore, because a feature may occur in any space of the image, the CNN is highly efficient and effective for image processing.

\subsection{Deep Q-Networks (DQNs)}

The DQN algorithm was developed by DeepMind in 2015. By combining reinforcement learning and deep neural networks on a large scale, we successfully solved a wide range of Atari games. The DQN is a combination of neural networks and reinforcement learning approaches; the connections between the nodes of a DQN exhibit a structure different from those of other network structures (Figure 2d). This was developed by improving the conventional reinforcement learning algorithm known as Q-learning using deep neural networks and a technique named experience replay DQNs can be categorized into two types of learning algorithm (e.g., Q-learning and deep Q-learning).

\section{ML-Based Urban Drainage System (UDS) Studies}

The following section presents a comprehensive review of recent ML-based UDS studies based on (1) operation (real-time operation control), (2) management (flood-inundation prediction), and (3) maintenance (pipe defect detection). To ensure a comprehensive review, Google Scholar, Scopus, and Web of Science were used to perform a search based on the aforementioned three topics. It is noteworthy that this review primarily focuses on methodologies, including the data types used, ML technology, and results obtained.

\subsection{Operation: Real-Time Operation Control}

This sub-section introduces real-time operation control based on reinforcement learning, which has emerged as a novel methodology for AI. Reinforcement learning is an ML field that pertains to the manner by which intelligent agents should perform actions in an environment to maximize the cumulative reward [30]. Reinforcement learning (see Figure 3) is one of three basic ML and/or DL paradigms, alongside supervised learning and 
unsupervised learning. It differs from supervised learning in that labeled input/output pairs are not presented, and sub-optimal actions need not be explicitly corrected. The environment is generally stated based on the Markov decision process approach, since other reinforcement learning algorithms in this context use dynamic programming techniques [31].

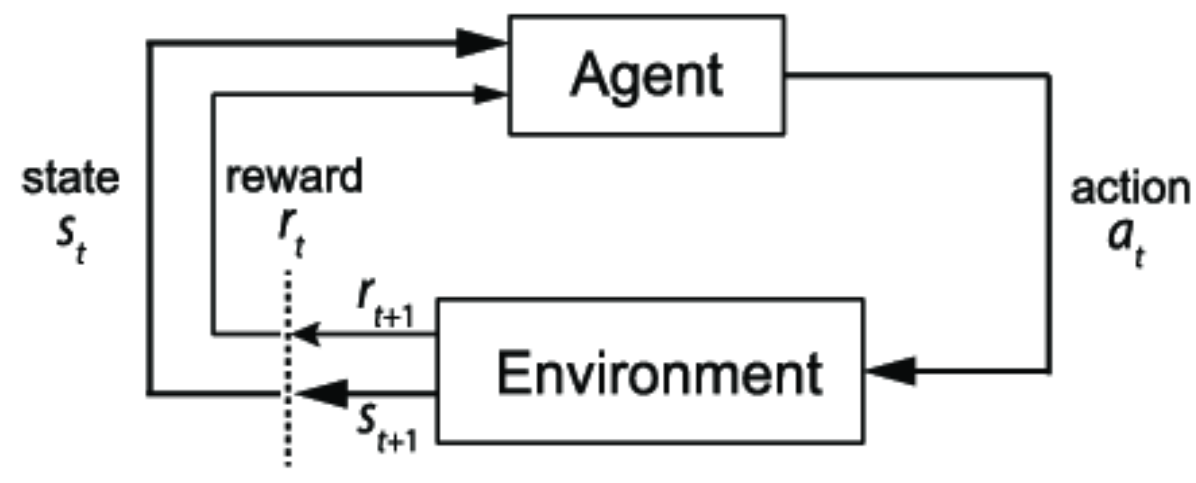

Figure 3. Schematic illustration of reinforcement learning.

Reinforcement learning can be derived as a Markov decision process approach of an agent combined with the environment to maximize the final reward. In Figure $1, t$ is the time step and $S_{t}$ is the current state (and current reward $r_{t}$ ). The agent must learn a value function that yields the optimal decision or action $a_{t}$. The action will affect the environment that induces the next reward signal $r_{t+1}$ and yields the next state $S_{t+1}$.

The UDS dynamically adapts its response to catastrophic rainfall by controlling its components, such as gates and pumps. Reinforcement learning has been widely applied recently by the AI community (e.g., robots and autonomous vehicles), whereas real-time operation control in UDSs is rarely implemented.

Several researchers [32,33] have proposed methodologies for real-time operation control in the UDS domain. Mullapudi et al. (2020) [32] formulated and analyzed a realtime operation control model (i.e., pumps) using reinforcement learning. Consequently, the automated operation system for UDSs demonstrated that reinforcement learning can effectively and efficiently pump operation and control for individual subcatchments. Wang et al. (2020) [34] performed pump operation control for a smart stormwater system using reinforcement learning to minimize flooding due to catastrophic rainfall events.

\subsection{Management: Flood-Inundation Prediction}

The purpose of flood-inundation prediction is to prevent damage to human life and properties. In UDSs, flood-inundation prediction can be defined as the process of estimating and predicting the magnitude and flood duration based on known characteristics. Flood-inundation prediction is the most important task in UDS modeling; hence end, ML technology has been used extensively to predict flood inundation.

To predict flood-inundation in UDSs, the ML technology such as ANN [35-48] and ANFIS [49] were primarily considered until the year 2010s. In the last decade, the time series analysis (e.g., wavelet decomposition, and fuzzy $[50,51]$ ) was mostly included with a flood inundation model based on the ML technology. Recently, wavelet decomposition is a time-series analysis approach that was used to predict flood inundation in the UDSs [52-54]. It was demonstrated that the technique can improve model performance from a solution of missing data problems [54].

The main contributions of these studies were to provide robustness, accuracy, effectiveness, and computation speed based on qualitative analysis and/or a flood-inundation prediction model. These studies faced significant limitations for improving the model performance (e.g., robustness, accuracy, effectiveness, and computation speed) more. To overcome the limitations, recently, the flood-inundation prediction studies have been 
performed primarily using ML technology, such as RNNs and CNNs. It is noteworthy that LSTM is a type of RNN. Table 1 summarizes relevant studies that focused on floodinundation prediction.

Table 1. Summary of studies pertaining to flood-inundation prediction by UDSs.

\begin{tabular}{cccc}
\hline References & Main Novelty & Input & ML Technology \\
\hline Xie et al. (2020) [55] & $\begin{array}{c}\text { Proposed ANN-based hybrid } \\
\text { modeling to improve model } \\
\text { performance }\end{array}$ & Inflow & ANN [20] \\
\hline $\begin{array}{c}\text { Hossein Hosseiny et al. } \\
\text { (2020) [56] }\end{array}$ & $\begin{array}{c}\text { Proposed novel hydraulics-ANN } \\
\text { hybrid model to identify flood area } \\
\text { and depth }\end{array}$ & $\begin{array}{c}\text { Water surface elevation, and } \\
\text { rough coefficient }\end{array}$ & ANN [20] \\
Kabir et al. (2020) [57] & $\begin{array}{c}\text { Proposed GPU-based CNN model } \\
\text { to improve model efficiency and } \\
\text { performance }\end{array}$ & $\begin{array}{c}\text { Discharge, and water depth } \\
\text { hydrograph }\end{array}$ & CNN [25] \\
\hline Guo et al. (2020) [58] & $\begin{array}{c}\text { Proposed image-to-image } \\
\text { translation to predict maximum } \\
\text { flood depth }\end{array}$ & Rainfall hyetograph & CNN [25] \\
\hline Ding et al. (2020) [59] & $\begin{array}{c}\text { Proposed flood forecasting and } \\
\text { interpretable spatiotemporal } \\
\text { attention LSTM }\end{array}$ & Rainfall, and inflow & LSTM (RNN) [23] \\
\hline Kao et al. (2020) [60] & $\begin{array}{c}\text { Proposed novel LSTM-based } \\
\text { encoder-decoder model for } \\
\text { multi-step-ahead flood-inundation } \\
\text { prediction }\end{array}$ & Rainfall, and inflow & \\
\hline
\end{tabular}

Flood-inundation prediction in the studies listed (Table 1) was performed using various ML techniques such as the ANN, CNN, and LSTM. In particular, the ANN and LSTM used are based on time-series data, which can estimate the flood-inundation results (e.g., flood depth and duration) for the next time step, although the spatial distribution of floods (e.g., flood area) is not provided. Meanwhile, the CNN is based on image data and can consider a spatiotemporal distribution for the type of flood-inundation (e.g., flood depth and area, and flood duration) prediction.

Kabir et al. (2020) [57] proposed a novel CNN model comprising a CNN based on a graphic processing unit to improve model efficiency (e.g., real-time flood forecasting) and performance simultaneously. Real-time-based flood-inundation forecasting models based on advanced measurements (e.g., drones and closed-circuit televisions (CCTVs)) have been proposed recently [60-63]. In summary, flood-inundation prediction models based on the CNN can be used for innovative real-time flood forecasting. However, the model's ability was not considered in some studies for solving complex problems involving large-scale datasets.

In addition, several flood-inundation prediction studies based on a physical model (e.g., one-dimensional (1D)/2D and 2D models) and ML techniques $[64,65]$ have been performed. Yang et al. (2019) [64] introduced an LSTM-based flood simulator coupled with a physical model to improve model performance. In their approach, the input data used were hydrological data, which included averaged daily precipitation, wind, temperature, and model-simulated discharge from the Global Hydrological Model + Catchment-based Macro-scale Floodplain between 1971 and 2020. Worland et al. (2019) [65] proposed a deep neural network comprising a rainfall-runoff model to estimate flood duration using hydrological data (e.g., discharge data) from the United States Geological Survey. It is noteworthy that the proposed model is a surrogate model based on a combination of a physically based model and ML technology. 


\subsection{Maintenance: Pipe Defect Detection}

A drainage pipe is a key component in UDSs. However, the quality of a drainage pipe deteriorates gradually owing to environmental and external conditions (e.g., ground subsidence). Therefore, defect detection in drainage pipes must be investigated.

In recent years, CCTV technologies have been widely used to effectively detect defects in drainage pipes used in UDSs. The required amount of image data can be obtained using CCTVs because UDSs are large. Error must be verified from the image data obtained from CCTVs; this process is time consuming and necessitates a practitioner. Therefore, pipe defect detection based on images obtained from CCTV has been primarily performed using ML technology in previous studies.

In ML techniques, the type of object detection has progressed rapidly. CNNs are widely used in image classification. In the last few decades, two trends have emerged from ML-based pipe defect detection models: (1) automatic feature classification from hybrid-based neural network [66-68]; (2) hierarchical feature classification using deep CNNs (e.g., fast R-CNN, faster R-CNN, You Only Look Once) [69-73]. The former is based on hybridizing the ANN and other ML technologies, whereas the latter is based on DL approaches.

For example, the R-CNN considers a selective search method to extract approximately 4000 regions from only one image as the input data for the $\mathrm{CNN}$ algorithm, whereas the fast R-CNN considers the convolutional feature map, which is focused on identifying detection. In addition, the faster R-CNN considers a region proposal network to perform region proposals and performs better than the fast R-CNN. Based on the aforementioned ML technologies, recent pipe defect detection studies pertaining to UDSs are summarized in Table 2.

Table 2. Summary of pipe defect detection studies pertaining to UDSs.

\begin{tabular}{|c|c|c|c|}
\hline References & Main Novelty & Defect Types & ML Techniques \\
\hline $\begin{array}{l}\text { Safari and Shoorehdeli } \\
\text { (2018) [74] }\end{array}$ & $\begin{array}{c}\text { Proposed detection of interior } \\
\text { defects based on image processing } \\
\text { and ANN }\end{array}$ & $\begin{array}{l}\text { Short, medium, and long } \\
\text { cracks; small, medium, and } \\
\text { large perforations }\end{array}$ & ANN [20] \\
\hline Kumar et al. (2018) [75] & $\begin{array}{l}\text { Proposed defect classification in } \\
\text { sewer CCTV inspections using deep } \\
\text { CNN }\end{array}$ & $\begin{array}{l}\text { Root intrusions, deposits, } \\
\text { cracks, infiltration, debris, } \\
\text { connections, and material } \\
\text { change }\end{array}$ & $\mathrm{CNN}$ [25] \\
\hline Cheng and Wang (2018) [76] & $\begin{array}{c}\text { Proposed detection of sewer pipe } \\
\text { defects based on CCTV images } \\
\text { using deep CNN }\end{array}$ & $\begin{array}{l}\text { Tree root intrusion, deposit, } \\
\text { infiltration, and cracks }\end{array}$ & $\mathrm{CNN}$ [25] \\
\hline Li and Guo (2019) [77] & $\begin{array}{l}\text { Proposed sewer damage detection } \\
\text { from imbalanced CCTV inspection } \\
\text { data using deep CNN with } \\
\text { hierarchical classification }\end{array}$ & $\begin{array}{c}\text { Deposit settlement, joint } \\
\text { offset, broken, obstacles, and } \\
\text { deformation }\end{array}$ & CNN [25] \\
\hline Yin et al. (2020) [78] & $\begin{array}{c}\text { Proposed deep CNN-based defect } \\
\text { detection system for sewer pipes } \\
\text { using CCTV }\end{array}$ & $\begin{array}{l}\text { Breakage, cracks, deposits, } \\
\text { fractures, taps, holes, and } \\
\text { roots }\end{array}$ & $\mathrm{CNN}$ [25] \\
\hline
\end{tabular}

As shown in Table 2, Safari and Shoorehdeli (2018) [74] proposed defect classification based on hybridization using an image-processing approach and an ANN. Other defect detection studies (based on Table 2) proposed performing defect classification and detection simultaneously to improve model performance and efficiency based on DL approaches (e.g., deep CNN). In terms of model performance, DL approaches are better than other ML technologies (e.g., ANN, SVM, and hybrid ML models). 


\section{Recommendations}

In this section, some research issues are presented, and recommendations for future ML-based UDS studies are provided. ML technology and/or DL approaches (e.g., CNN and RNN) require large sample sizes (i.e., big data). Although the studies presented in Section 2 used small-scale data (e.g., single size) only, only DL approaches were applied, whereas the model efficiency for a complex model architecture based on large-scale datasets was not considered. Hence, the advantages increased with the data size.

In addition, ML-based applications in UDSs have increased rapidly owing to their high-performance computing power, as well as their ability to process big data and perform AI. Publications pertaining to the application of ML in UDSs abound. However, ML-based UDSs are associated with two main challenges, and overcoming these challenges will further improve their performance and efficiency.

\subsection{Challenges}

First, theoretical problems in ML-based UDS modeling are primarily considered in terms of two aspects (i.e., mathematics and computation capability). In any non-linear function, the types of ML-based neural networks (e.g., shallow networks) and neural networks based on DL approaches (e.g., deep networks) can be represented. It is discovered that DL approaches yield better model performances than the former (i.e., non-linear function-based neural networks). However, the representation and computation capability of DL approaches does not imply that they are better than non-linear function networks. For ML-based UDSs, the complexity of data samples must be understood, and the number of learning data samples required for learning the DL approach types as well as the computing performance required for the training data samples must be determined. In addition, DL approaches generally involve non-convex functions, and it is theoretically complex to train the neural network and optimize numerous parameters based on DL.

Next, most studies pertaining to ML-based UDSs that consider large-scale input data have shown that complex DL approaches are more suitable for obtaining significant features. The features and other information extracted from large-scale data samples are meaningful. The essence of DL approaches is to train more significant features directly and ultimately improve the model performance and efficiency. When comparing shallow and deep networks, DL approaches emphasize the important characteristics because the DL model contains hidden layers of various types (e.g., five to 10 layers). DL approaches provide an efficient model through layer-by-layer feature learning from the image or timeseries data related to UDS modeling. However, constructing a hierarchical model with effective learning to identify important features is a key issue. In addition, selecting the most suitable ML-based UDS modeling is important.

Finally, the performance of an ML-based model varies significantly depending on whether an observation dataset is available. In a previous study, for example, a floodinundation prediction model based on image data was limited in terms of comparing results obtained from a physically based hydrodynamic/hydraulic model (e.g., 1D/2D and 2D models) to verify the model performance. In other words, the model's performance does not agree well with the results obtained from the physically based hydrodynamic/hydraulic models because the observation data do not include the flood area and depth (actually measured data). Hence, the model's performance must be improved significantly based on observation data, e.g., flood area and depth, obtained accurately using advanced equipment such as satellites and drones.

\subsection{Future Directions}

Based on this comprehensive review, the authors recommend the following future directions for studies pertaining to ML-based UDSs:

(1). Feature extraction method: the efficient identification of features from advanced measurements (e.g., CCTVs and drones) that comprise only a single DL algorithm 
(e.g., CNN) can be considered. The integration of a hybrid DL algorithm (e.g., RNNCNN) should be considered to effectively extract important features.

(2). Computation resources and combination with physically based model: the processing of physically based hydrodynamic/hydraulic models (e.g., 1D/2D and 2D model) is time consuming. Such limitations are caused by the fact that real-time prediction, operation, and simulation cannot be considered. Therefore, ML-based UDS studies that actively consider high-performance computing resources (e.g., graphic processing units and supercomputers) should be conducted.

(3). Necessity to increase utilization of reinforcement learning: the development of UDSs based on reinforcement learning is rare. However, reinforcement learning can be applied and considered in terms of the UDS operation, e.g., the gate and/or pump operation in the UDS. In addition, a practitioner should refer to a predefined manual to control the gate or pump operation. Therefore, considering reinforcement learningbased UDSs for pump and/or gate operations can reduce mistakes from practitioners.

(4). Necessity to utilize advanced technologies (satellites and drones): advanced technologies such as analyses and predictions based on raw data using satellites and drones, which are advanced equipment based on remote-sensing fields, should be developed. In addition, a new and/or advanced methodology for improving the predictive power of water information presented as an image, as well as the time-series form, should be developed to analyze the characteristics and patterns of each element. ML technology must be systematically used to develop such a methodology. In the case of ML technology, because it is a data-based approach, a significant amount of data and high quality must be ensured for its advancement. In particular, multiple remote sensors based on the cloud and Internet of Things should be implemented in UDSs such that a significant amount of water information (e.g., precipitation, temperature, wind, humidity, flow rate, and water level) can be acquired.

By realizing the recommended implementations above, analysis and prediction technologies can be further developed to realize better system operation, management, maintenance, and risk standards for UDSs.

\section{Conclusions}

In the recent decade, ML techniques have been transforming daily lives, industries, and various scientific/engineering disciplines. In particular, ML approaches have afforded significant progress in neural network models, thereby enabling the automatic computation of problem-relevant features and rapid capture of highly complex data distributions. ML approaches can address several significant new and/or old challenges pertaining to UDSs. This review paper provides a comprehensive review of recent UDS modeling based on ML technology, including DL. We believe that ML technology can address several significant new and/or old challenges facing research in the UDS domain. This comprehensive review classifies studies pertaining to ML-based UDSs into three research categories: operation (real-time operation control), management (flood-inundation prediction), and maintenance (pipe defect detection).

Finally, several recommendations for future studies were presented herein. Understanding the main characteristics of ML technology enables efficient and effective UDS modeling, thereby enabling the development of a system that can prepare and respond to catastrophic rainfall events. Some potential issues and future directions are recommended for each of the three research topics to extend UDS modeling/applications based on ML approaches. In addition, ML approaches can promote advances in UDSs significantly.

The new paradigm of ML-based UDS modeling summarized herein is still in its early stage and should be considered in future studies. In summary, UDS studies based on ML technology that involve operation, management, and maintenance must be further conducted such that features can be developed more efficiently, i.e., computation resources (i.e., graphic processing units and supercomputers) and advanced equipment (i.e., satellites 
and drones) should be considered effectively such that multidisciplinary and broader analyses can be realized.

Author Contributions: Conceptualization, S.H.K.; Investigation, S.H.K.; Methodology, S.H.K.; Project administration, J.H.K.; Supervision, J.H.K.; Visualization, S.H.K.; Writing-original draft preparation, S.H.K.; Writing - review \& editing, and J.H.K. All authors have read and agreed to the published version of the manuscript.

Funding: This work was supported by a National Research Foundation of Korea (NRF) grant funded by the Korean government (Ministry of Science and ICT (MSIT)) (No. 2019R1A2B5B03069810).

Institutional Review Board Statement: Not applicable.

Informed Consent Statement: Not applicable.

Data Availability Statement: Not applicable.

Conflicts of Interest: The authors declare no conflict of interest.

$\begin{array}{ll}\text { Abbreviation } \\ \text { ML } & \text { Machine learning } \\ \text { DL } & \text { Deep learning } \\ \text { UDS } & \text { Urban drainage system } \\ \text { AI } & \text { Artificial intelligence } \\ \text { ANN } & \text { Artificial neural network } \\ \text { CNN } & \text { Convolutional neural network } \\ \text { RNN } & \text { Recurrent neural network } \\ \text { DQN } & \text { Deep Q-network } \\ \text { LSTM } & \text { Long short-term memory } \\ \text { CCTV } & \text { Closed-circuit television } \\ \text { IoT } & \text { Internet of Things }\end{array}$

\section{References}

1. Hsu, M.H.; Chen, S.H.; Chang, T.J. Inundation simulation for urban drainage basin with storm sewer system. J. Hydrol. 2000, 234, 21-37. [CrossRef]

2. Abderrezzak, K.E.K.; Paquier, A.; Mignot, E. Modelling flash flood propagation in urban areas using a two-dimensional numerical model. Nat. Hazards 2009, 50, 433-460. [CrossRef]

3. Sanudo, E.; Cea, L.; Puertas, J. Modelling Pluvial Flooding in Urban Areas Coupling the Models Iber and SWMM. Water 2020, 12, 2647. [CrossRef]

4. Patowary, S.; Sarma, A.K. Two-Dimensional Numerical Model for Urban Drainage System. Urban Hydrol. $2016,73,163-173$.

5. Bhola, P.K.; Leandro, J.; Disse, M. Framework for Offline Flood Inundation Forecasts for Two-Dimensional Hydrodynamic Models. Geosiciences 2018, 8, 346. [CrossRef]

6. Keum, H.J.; Han, K.Y.; Kim, H.Y. Real-Time Flood Disaster Prediction System by Applying Machine Learning Technique. Water Resour. Hydrol. Eng. 2020, 24, 2835-3848. [CrossRef]

7. Bates, P.D.; Horritt, M.S.; Fewtrell, T.J. A simple inertial formulation of the shallow water equations for efficient two-dimensional flood inundation modelling. J. Hydrol. 2010, 387, 33-45. [CrossRef]

8. Kalyanapu, A.J.; Shanker, S.; Pardyjak, E.R.; Judi, D.R.; Burian, S.J. Assessment of GPU computational enhancement to a 2D flood model. Environ. Model. Softw. 2011, 26, 1009-1016. [CrossRef]

9. Munawar, H.S.; Hammad, A.W.A.; Waller, S.T. A review on flood management technologies related to image processing and machine learning. Autom. Constr. 2021, 132, 103916. [CrossRef]

10. Mosavi, A.; Ozturk, P.; Chau, K. Flood Prediction Using Machine Learning Models: Literature Review. Water 2018, $10,1536$. [CrossRef]

11. Bui, D.T.; Hoaug, N.; Martínez-Álvarez, F.; Ngo, P.T.; Hoa, P.V.; Pham, T.D.; Samui, P.; Costache, R. A novel deep learning neural network approach for predicting flash flood susceptibility: A case study at a high frequency tropical storm area. Sci. Total Environ. 2020, 20, 134413.

12. Wadiasari, I.R.; Nugroho, L.E. Deep learning multilayer perceptron (MLP) for flood prediction model using wireless sensor network based hydrology time series data mining. In Proceedings of the International Conference on Innovative and Creative Information Technology (ICITech), Salatiga, Indonesia, 2-4 November 2017.

13. Dong, S.; Yu, T.; Farahmand, H.; Mostafavi, A. A hybrid deep learning model for predictive flood warning and situation awareness using channel network sensors data. Comput.-Aided Civ. Infrastruct. Eng. 2020, 36, 402-420. [CrossRef] 
14. Hu, R.; Fang, F.; Pain, C.C.; Navon, I.M. Rapid spatio-temporal flood prediction and uncertainty quantification using a deep learning method. J. Hydrol. 2019, 575, 911-920. [CrossRef]

15. Song, T.; Ding, W.; Liu, H.; Wu, J.; Zhou, H.; Chu, J. Uncertainty Quantification in Machine Learning Modeling for Multi-Step Time Series Forecasting: Example of Recurrent Neural Networks in Discharge Simulations. Water 2020, 12, 912. [CrossRef]

16. Sankaranarayanan, S.; Prabhakar, M.; Satish, S.; Jain, P.; Ramprasad, A.; Krishnan, A. Flood prediction based on weather parameters using deep learning. J. Water Clim. Chang. 2020, 11, 1766-1783. [CrossRef]

17. Saba, L.; Biswas, M.; Kuppili, V.; Godia, E.C.; Suri, H.S.; Edla, D.R.; Omerzu, T.; Laird, J.R.; Khanna, N.N.; Mavrogeni, S.; et al. The present and future of deep learning in radiology. Eur. J. Radiol. 2019, 114, 14-24. [CrossRef] [PubMed]

18. Sahiner, B.; Pezeshk, A.; Hadjiiski, L.M.; Wang, X.; Drukker, K.; Cha, K.H.; Summers, R.M.; Giger, M.L. Deep learning in medical imaging and radiation therapy. Med. Phys. 2019, 46, e1-e36. [CrossRef] [PubMed]

19. Ochoa, D.; Riano-Briceno, G.; Quijano, N.; Ocampo-Martinez, C. Control of Urban Drainage Systems: Optimal Flow Control and Deep Learning in Action. In Proceedings of the American Control Conference (ACC), Philadelphia, PA, USA, 10-12 July 2019.

20. Werbos, P. Beyond Regression: New Tools for Prediction and Analysis in the Behavioral Sciences. Ph.D. Dissertation, Harvard University, Cambridge, MA, USA, 1974.

21. Dupond, S. A thorough review on the current advance of neural network structures. Annu. Rev. Control 2019, 14, $200-230$.

22. Abiodun, O.I.; Jantan, A.; Omolara, A.E.; Dada, K.V.; Mohamed, N.A.; Arshad, H. State-of-the-art in artificial neural network applications: A survey. Heliyon 2018, 4, e00938. [CrossRef]

23. Tealab, A. Time series forecasting using artificial neural networks methodologies: A systematic review. Future Comput. Inform. J. 2018, 3, 334-340. [CrossRef]

24. Sepp, H.; Jürgen, S. Long short-term memory. Neural Comput. 1997, 9, 1735-1780.

25. Hubel, D.H.; Wiesel, T.N. Receptive fields and functional architecture of monkey striate cortex. J. Physiol. 1968, 195, 215-243. [CrossRef] [PubMed]

26. Fukushima, K.; Miyake, S. Neocognitron: A self organizing neural network model for a mechanism of visual pattern recognition. In Competition and Cooperation in Neural Nets; Springer: Berlin, Germany, 1982; pp. 267-285.

27. Valueva, M.V.; Nagornov, N.N.; Lyakhov, P.A.; Valuev, G.V.; Chervyakov, N.I. Application of the residue number system to reduce hardware costs of the convolutional neural network implementation. Math. Comput. Simul. 2020, 177, 232-243. [CrossRef]

28. Zhang, W. Shift-invariant pattern recognition neural network and its optical architecture. In Proceedings of the Annual Conference of the Japan Society of Applied Physics, Tokyo, Japan, 9-13 September 2018.

29. Zhang, W. Parallel distributed processing model with local space-invariant interconnections and its optical architecture. Appl. Opt. 1990, 29, 4790-4797. [CrossRef] [PubMed]

30. Hu, J.; Niu, H.; Carrasco, J.; Lennox, B.; Arvin, F. Voronoi-Based Multi-Robot Autonomous Exploration in Unknown Environments via Deep Reinforcement Learning. IEEE Trans. Veh. Technol. 2020, 69, 14413-14423. [CrossRef]

31. Otterlo, M.V.; Wiering, M. Reinforcement learning and markov decision processes. In Reinforcement Learning; Springer: Berlin, Germany, 2012.

32. Mullapudi, A.; Lewis, M.J.; Gruden, C.L.; Kerkez, B. Deep reinforcement learning for the real time control of stormwater systems. Adv. Water Resour. 2020, 140, 103600. [CrossRef]

33. Martinez-Piazuelo, J.; Ochoa, D.E.; Quijano, N.; Giraldo, L.F. A multi-critic reinforcement learning method: An application to multi-tank water systems. IEEE Access. 2020, 8, 173227-173238. [CrossRef]

34. Wang, C.; Bowes, B.; Tavakoli, A.; Adams, S.; Goodall, J.; Beling, P. Smart Stormwater Control Systems: A Reinforcement Learning Approach. In Proceedings of the ISCRAM Conference Proceedings-17th International Conference on Information Systems for Crisis Response and Management, Blacksburg, VA, USA, 24-27 May 2020.

35. Kim, S.; Singh, V.P. Flood forecasting using neural computing techniques and conceptual class segregation. J. Am. Water Resour. Assoc. 2013, 49, 1421-1435. [CrossRef]

36. Jingyi, Z.; Hall, M.J. Regional flood frequency analysis for the Gan-Ming River basin in China. J. Hydrol. 2004, $296,98-117$. [CrossRef]

37. Sahoo, G.B.; Ray, C.; De Carlo, E.H. Use of neural network to predict flash flood and attendant water qualities of a mountainous stream on Oahu, Hawaii. J. Hydrol. 2006, 327, 525-538. [CrossRef]

38. Chang, F.-J.; Chen, P.-A.; Lu, Y.-R.; Huang, E.; Chang, K.-Y. Real-time multi-step-ahead water level forecasting by recurrent neural networks for urban flood control. J. Hydrol. 2014, 517, 836-846. [CrossRef]

39. Shen, H.-Y.; Chang, L.-C. Online multistep-ahead inundation depth forecasts by recurrent NARX networks. Hydrol. Earth Syst. Sci. 2013, 17, 935-945. [CrossRef]

40. Heiser, M.; Scheidl, C.; Eisl, J.; Spangl, B.; Hübl, J. Process type identification in torrential catchments in the eastern Alps. Geomorphology 2015, 232, 239-247. [CrossRef]

41. Wei, C.C. Soft computing techniques in ensemble precipitation nowcast. Appl. Soft Comput. J. 2013, 13, 793-805. [CrossRef]

42. Chang, L.-C.; Shen, H.-Y.; Chang, F.-J. Regional flood inundation nowcast using hybrid SOM and dynamic neural networks. J. Hydrol. 2014, 519, 476-489. [CrossRef]

43. French, J.; Mawdsley, R.; Fujiyama, T.; Achuthan, K. Combining machine learning with computational hydrodynamics for prediction of tidal surge inundation at estuarine ports. Proced. IUTAM 2017, 25, 28-35. [CrossRef]

44. Hong, W.-C. Rainfall forecasting by technological machine learning models. Appl. Math. Comput. 2008, 200, 41-57. [CrossRef] 
45. Rajurkar, M.; Kothyari, U.; Chaube, U. Modeling of the daily rainfall-runoff relationship with artificial neural network. J. Hydrol. 2004, 285, 96-113. [CrossRef]

46. Hsu, M.-H.; Lin, S.-H.; Fu, J.-C.; Chung, S.F.; Chen, A.S. Longitudinal stage profiles forecasting in rivers for flash floods. J. Hydrol. 2010, 388, 426-437. [CrossRef]

47. Fleming, S.W.; Bourdin, D.R.; Campbell, D.; Stull, R.B.; Gardner, T. Development and operational testing of a super-ensemble artificial intelligence flood-forecast model for a pacific northwest river. J. Am. Water Resour. Assoc. 2015, 51, 502-512. [CrossRef]

48. Xie, S.; Wu, W.; Mooser, S.; Wang, Q.J.; Nathan, R.; Huang, Y. Artificial neural network based hybrid modeling approach for flood inundation modeling. J. Hydrol. 2021, 592, 125605. [CrossRef]

49. Jimeno-Sáez, P.; Senent-Aparicio, J.; Pérez-Sánchez, J.; Pulido-Velazquez, D.; Cecilia, J.M. Estimation of instantaneous peak flow using machine-learning models and empirical formula in peninsular Spain. Water 2017, 9, 347. [CrossRef]

50. Shu, C.; Ouarda, T. Regional flood frequency analysis at ungauged sites using the adaptive neuro-fuzzy inference system. J. Hydrol. 2008, 349, 31-43. [CrossRef]

51. Lohani, A.K.; Goel, N.; Bhatia, K. Improving real time flood forecasting using fuzzy inference system. J. Hydrol. 2014, 509, 25-41. [CrossRef]

52. Ghaderpour, E.; Vujadinovic, T.; Hassan, Q.K. Application of the least-squares wavelet software in hydrology: Athabasca River basin. J. Hydrol. Reg. Stud. 2021, 36, 100847. [CrossRef]

53. Altunkaynak, A.; Nigussie, T.A. Prediction of daily rainfall by a hybrid wavelet-season-neuro technique. J. Hydrol. 2015, 529, 287-301. [CrossRef]

54. Yang, M.; Sang, Y.F.; Liu, C.; Wang, Z. Discussion on the choice of decomposition level for wavelet based hydrological time series modeling. Water 2016, 8, 197. [CrossRef]

55. Hosseiny, H.; Nazari, F.; Smith, V.; Nataraj, C. A framework for modeling flood depth using a hybrid of hydraulics and machine learning. Sci. Rep. 2020, 10, 8222. [CrossRef] [PubMed]

56. Kabir, S.; Patidar, S.; Xia, X.; Liang, Q.; Neal, J.; Pender, G. A deep convolutional neural network model for rapid prediction of fluvial flood inundation. J. Hydrol. 2020, 590, 125481. [CrossRef]

57. Guo, Z.; Leitao, J.P.; Simões, N.E.; Moosavi, V. Data-driven flood emulation: Speeding up urban flood predictions by deep convolutional neural networks. J. Flood Risk Manag. 2021, 14, 12684. [CrossRef]

58. Ding, Y.; Zhu, Y.; Feng, J.; Zhang, P.; Cheng, Z. Interpretable spatio-temporal attention LSTM model for flood forecasting. Neurocomputing 2020, 403, 348-359. [CrossRef]

59. Kao, I.F.; Zhou, Y.; Chang, L.C.; Chang, F.J. Exploring a Long Short-Term Memory based Encoder-Decoder framework for multi-step-ahead flood forecasting. J. Hydrol. 2020, 583, 124631. [CrossRef]

60. Wang, J.H.; Lin, G.F.; Chang, M.J.; Huang, I.H.; Chen, Y.R. Real-time water-level forecasting using dilated causal convolutional neural networks. Water Resour. Manag. 2019, 33, 3759-3780. [CrossRef]

61. Bhola, P.K.; Nair, B.B.; Leandro, J.; Rao, S.N.; Disse, M. Flood inundation forecasts using validation data generated with the assistance of computer vision. J. Hydroinform. 2019, 21, 240-256. [CrossRef]

62. Moy de Vitry, M.; Kramer, S.; Wegner, J.D.; Leitão, J.P. Scalable flood level trend monitoring with surveillance cameras using a deep convolutional neural network. Hydrol. Earth Syst. Sci. 2019, 23, 4621-4634. [CrossRef]

63. Bermúdez, M.; Cea, L.; Puertas, J. A rapid flood inundation model for hazard mapping based on least squares support vector machine regression. J. Flood Risk Manag. 2019, 12, 12522. [CrossRef]

64. Yang, T.; Sun, F.; Gentine, P.; Liu, W.; Wang, H.; Yin, J.; Du, M.; Liu, C. Evaluation and machine learning improvement of global hydrological model-based flood simulations. Environ. Res. Lett. 2019, 14, 114027. [CrossRef]

65. Worland, S.C.; Steinschneider, S.; Asquith, W.; Knight, R.; Wieczorek, M. Prediction and inference of flow duration curves using multioutput neural networks. Water Resour. Res. 2019, 55, 6850-6868. [CrossRef]

66. Moselhi, O.; Shehab-Eldeen, T. Automated detection of surface defects in water and sewer pipes. Autom. Constr. 1999, 8, 581-588. [CrossRef]

67. Moselhi, O.; Shehab-Eldeen, T. Classification of defects in sewer pipes using neural networks. J. Infrastruct. Syst. 2000, 6, 97-104. [CrossRef]

68. Shehab, T.; Moselhi, O. Automated detection and classification of infiltration in sewer pipes. J. Infrastruct. Syst. 2005, 11, 165-171. [CrossRef]

69. Girshick, R.; Donahue, J.; Darrell, T.; Malik, J. Rich feature hierarchies for accurate object detection and semantic segmentation. In Proceedings of the IEEE Conference on Computer Vision and Pattern Recognition, Columbus, OH, USA, $24-27$ June 2014.

70. Girshick, R. Fast R-CNN. In Proceedings of the IEEE International Conference on Computer Vision, Santiago, Chile, 7-13 December 2015.

71. Chen, S.; Mulgrew, B.; Grant, P.M. A clustering technique for digital communications channel equalization using radial basis function networks. IEEE Trans. Neural Netw. 1993, 4, 570-590. [CrossRef] [PubMed]

72. Demirkol, A.; Demir, Z.; Emre, E. Lecture Notes in Computer Science; Springer: Berlin, Germany, 2005.

73. Redmon, J.; Divvala, S.; Girshick, R.; Farhadi, A. You only look once: Unified, real-time object detection. In Proceedings of the IEEE Conference on Computer Vision and Pattern Recognition, Las Vegas, NV, USA, 26 June-1 July 2016.

74. Safari, S.; Aliyari, S.M. Detection and isolation of interior defects based on image processing and neural networks: HDPE pipeline case study. J. Pipeline Syst. Eng. Pract. 2018, 9, 05018001. [CrossRef] 
75. Kumar, S.S.; Abraham, D.M.; Jahanshahi, M.R.; Iseley, T.; Starr, J. Automated defect classification in sewer closed circuit television inspections using deep convolutional neural networks. Autom. Constr. 2018, 91, 273-283. [CrossRef]

76. Cheng, J.C.; Wang, M. Automated detection of sewer pipe defects in closed-circuit television images using deep learning techniques. Autom. Constr. 2018, 95, 155-171. [CrossRef]

77. Li, D.; Cong, A.; Guo, S. Sewer damage detection from imbalanced CCTV inspection data using deep convolutional neural networks with hierarchical classification. Autom. Constr. 2019, 101, 199-208. [CrossRef]

78. Yin, X.; Chen, Y.; Bouferguene, A.; Zaman, H.; Al-Hussein, M.; Kurach, L. A deep learning-based framework for an automated defect detection system for sewer pipes. Autom. Constr. 2020, 109, 102967. [CrossRef] 